\title{
Reconstitution of Tubulin Methylation by SETD2
}

\author{
Sarah Kearns ${ }^{1,2^{*}}$, Michael Cianfrocco ${ }^{2,3}$, and Kristen Verhey ${ }^{4}$ \\ 1. Chemical Biology Program, University of Michigan, Ann Arbor, USA. \\ 2. Life Sciences Institute, University of Michigan, Ann Arbor, USA. \\ 3. Department of Biological Chemistry, University of Michigan, Ann Arbor, USA. \\ 4. Department of Cellular and Developmental Biology, University of Michigan, Ann Arbor, USA. \\ * Corresponding author: skearns@umich.edu
}

Microtubules form one of the major components of the eukaryotic cytoskeleton. Assembled from evolutionarily conserved dimers of alpha and beta tubulin, microtubules are involved in a wide variety of cellular functions that range from transporting cargo to forming the mitotic spindle and creating primary cilia and flagella. Behaviour and function of microtubules are affected by their physical properties such as switching between growth and shrinkage (termed dynamic instability), and interacting with a range of microtubule associated proteins [1]. Even though microtubules are made of the same heterodimer building blocks, distinct populations localize in cells for specific functions including spatial organization, directional transport, and force generation. How microtubules achieve this functional diversity is at least in part driven by a "Tubulin Code" of tubulin isotypes and post-translational modifications (PTMs) (Figure 1). Analogous to the "Histone Code," cells leverage the Tubulin Code to construct microtubule architecture with particular dynamical properties and specific functions [2]. The human genome encodes 8 alpha and 9 beta isotypes which have varying expression levels and functional properties. Most of these sequence differences in isotypes come from tubulin's dynamic C-terminal tails (CTTs). Additionally, many known PTMs are found on these CTTs and have cellular localization and functional output in cells. To crack the PTM component of the Tubulin Code, both the modification and its writer need to be determined and characterized. So far, PTMs including, but not limited to, detyrosination, polyglutamylation, and acetylation are found enriched on specialized microtubule structures and their chemical writers and erasers have been identified [3] (Figure 1E).

Recently, methylation has joined the ranks as a tubulin modification, occurring on spindle and midbody microtubules during mitosis [4]. Through a combination of mass spectrometry, co- immunoprecipitation, and immunofluorescence, histone lysine methyltransferase SETD2 was found to bind and methylate tubulin at the alpha-K40 site, the same cite as acetylation [5]. To examine the impact of SETD2 during mitosis, an inducible knockout cell line was produced and viewed with live-cell imaging. The resulting phenotype displayed mitotic spindle and cytokinesis defects, chromosomal bridging, and micronuclei formation, all drivers of cancer. A series of rescue experiments with pathogenic SETD2 constructs suggests that the defects are histone independent, where a particular SETD2 mutation that does not diminish histone methylation activity dramatically impairs tubulin activity. Interestingly, these mutations are implicated in many cancers, especially in the most common form of kidney cancer clear cell renal cell carcinoma $[6,7]$.

To investigate SETD2's tubulin methylation activity and cellular output, we are combining biochemical, kinetic, single-molecule, and structural studies. An active truncated construct of SETD2, which contains the catalytic SET domain to the C-terminal SRI domain (Figure 2A), methylates both histone and tubulin. A cancer-driving mutation in the SET domain ablates all SETD2 activity regardless of substrate, but the aforementioned histone-independent mutation, found in the SRI domain, reduces 
tubulin methylation confirming previous in vivo rescue experiments (Figure 2B). In a histone-modifying context, the SRI domain is responsible for making contacts with phosphorylated RNA Polymerase [8]. Performing mass spectrometry on both endogenous tubulin and SETD2-modified recombinant tubulin have identified sites of methylation, indicating that we have reconstituted in vitro methylation of tubulin. Additionally, our work suggests that SETD2 methylation may preferentially occur occur on tubulin dimers instead of polymerized microtubules. This provides a target for structural studies using cryo- electron microscopy. Subsequent structure determination will elucidate how the histone methyltransferase SETD2 is able to recognize and modify tubulin in an SRI-domain dependent way, and how methylation impacts the cytoskeletal and mitotic spindle structure. Deletion of the SRI-domain of SETD2 or tubulin CTT will also highlight the importance components for substrate recognition. Single molecule and fluorescence in vivo studies tracking methylated tubulin will additionally determine the role of methylated tubulin, or lack thereof, within a cancer phenotype context.

\section{References:}

[1] M Kirschner and T Mitchison, Cell 45 (1986), p. 329.

[2] K Verhey and J Gaertig, Cell Cycle 6 (2007), p. 2152.

[3] M Magiera and C Janke, Cell 173(6) (2018), p. 1552.

[4] I Park and C Walker, Cell 166(4) (2017), p. 950.

[5] V Soppina and K Verhey, PLoS ONE 7 (2012), e48204.

[6] Y Chiang and W Rathmell, Cancer Research. 78(12) (2018), p. 3135.

[7] K Hacker and W Rathmell, JBC 291(40) (2016), p. 21283.

[8] M Li and P Zhou, PNAS 102(49) (2005), p. 17636.
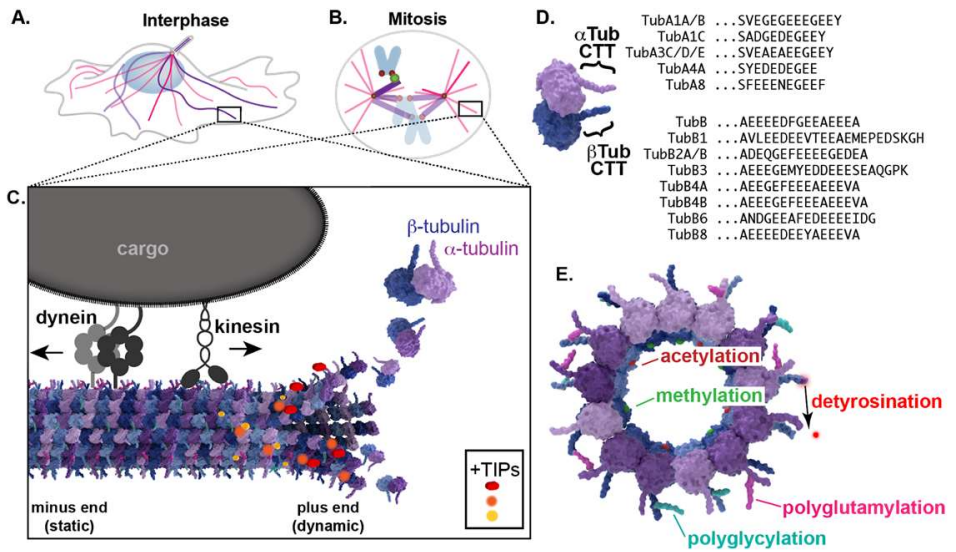

Figure 1. Tubulin Code.

\begin{tabular}{|c|c|c|c|c|c|}
\hline A & AWS & SET & $\begin{array}{l}\text { oost- } \\
\text { SET }\end{array}$ & & SRI \\
\hline \multirow[t]{4}{*}{ B } & & tivity & H3 peptide & Tubulin & \\
\hline & & T SETD2 & Yes & Yes & \\
\hline & & $1625 \mathrm{C}$ & No & No & \\
\hline & & $2510 G$ & Yes & No & \\
\hline
\end{tabular}

Figure 2. SETD2 activity. A) Domain lay-out of active truncated SETD2 construct. B) Methylation activity based on fluorescence assay of WT and mutant SETD2, mutations color coded to domain. 